\title{
Adipocytic Progenitor Cells Give Rise to Pathogenic Myofibroblasts: Adipocyte-to-Mesenchymal Transition and Its Emerging Role in Fibrosis in Multiple Organs
}

\author{
Roberta Goncalves Marangoni ${ }^{1}$ (D) B Benjamin Korman ${ }^{1} \cdot J^{\prime}$ ohn Varga ${ }^{2}$ \\ Published online: 25 September 2020 \\ (C) Springer Science+Business Media, LLC, part of Springer Nature 2020
}

\begin{abstract}
Purpose of Review Adipocytes have recently been shown to be able to reprogram to a myofibroblastic phenotype in a process termed adipocyte mesenchymal transition (AMT). This review seeks to discuss the relevance of this process to disease and explore its mechanisms.

Recent Findings AMT occurs in multiple organs and diseases, transdifferentiation goes through a precursor cell and there is a reversible process that can be influenced by metabolic stress, myeloid cells, immune dysregulation, and pharmacological intervention.

Summary AMT is a newly appreciated and highly relevant process in multiple forms of fibrosis. Targeting AMT may serve as a novel method of treating fibrosis.
\end{abstract}

Keywords Fibroblast $\cdot$ Myofibroblast $\cdot$ Adipocyte $\cdot$ Fibrosis $\cdot$ Scleroderma $\cdot$ Adipose mesenchymal transition

\section{Introduction}

Fibroblasts and myofibroblasts are tissue-resident mesenchymal cells that serve as the main effectors in all forms of fibrosis, contributing to excessive scarring and organ dysfunction in a diverse group of chronic conditions [1]. Given their central role, fibroblasts and myofibroblasts have for two decades been the primary focus of studies seeking to understand the pathogenesis of fibrosis and the development of targeted anti-

This article is part of the Topical Collection on Scleroderma

Roberta Goncalves Marangoni

Roberta_Goncalvesmarangoni@urmc.rochester.edu

Benjamin Korman

benjamin_korman@urmc.rochester.edu

John Varga

j-varga@northwestern.edu

1 Division of Allergy, Immunology and Rheumatology, University of Rochester Medical Center, 601 Elmwood Avenue BOX 695, Rochester, NY 14642, USA

2 Division of Rheumatology, Northwestern University Feinberg School of Medicine, 240 E. Huron Street, McGaw Pavilion M-300, Chicago, IL 60611, USA fibrotic therapies. In this review, we will summarize recent progress regarding the cellular origins of fibroblasts in fibrotic states, and emerging evidence implicating a novel mechanism of adipocyte-derived myofibroblast differentiation as an important and therapeutically tractable mechanism in fibrosis in systemic sclerosis (SSc) and other diseases associated with fibrosis.

\section{Fibroblasts and Myofibroblasts}

Fibroblasts are a heterogeneous group of tissue-resident mesenchymal cells. Fibroblasts produce collagen, are not terminally differentiated, and, significantly, lack uniform cell surface markers [2]. Multiple factors have been identified as triggers for fibroblast activation and important in disease pathogenesis. These include transforming growth factor- $\beta$ (TGF- $\beta$ ), IL-11, platelet-derived growth factor (PDGF), Wnt, hedgehog, and YAP-TAZ signaling [3]. When activated, fibroblasts undergo extensive reprogramming and transition to a myofibroblast phenotype. Myofibroblasts express stress fibers giving them the ability to exert contractile forces in the extracellular matrix (ECM). These contractile forces along with excessive accumulation of collagen and other ECM macromolecules that comprise the matrisome lead to rising tissue 
stiffness which activates mechanosensors and intracellular mechanotransduction pathways, creating self-amplifying fibrotic loops [4]. As opposed to the tightly regulated physiologic process that occurs in normal wound healing, in fibrosis, fibroblasts evade apoptosis and persist in a chronically activated state [5].

The origin of myofibroblasts in fibrotic tissue has been the subject of intensive investigation but to date remains controversial. Multiple contributing cells, mostly in the perivascular niche, have been implicated but uncertainty remains as to the main contributors [6]. While tissueresident fibroblasts are often thought to be a major precursor, lineage-tracing studies in mice have demonstrated transdifferentiation or reprogramming from a number of important non-hematopoietic cells types including epithelial and endothelial cells, pericytes, as well as various populations of bone marrow-derived cells [7]. Epithelial-mesenchymal transition (EMT), a normal physiological process involved in organogenesis during embryonic development, has been documented in a number of fibrotic diseases, particularly those affecting the lung and kidney [8]. In this process, epithelial cells such as alveolar pneumocytes lose their epithelial markers and tight junctions and differentiate into an invasive mesenchymal phenotype with the ability to migrate [9]. More recently, it has become apparent that vascular endothelial cells can similarly switch their phenotype during fibrogenesis. They do this by downregulating intercellular adhesion molecules, such as cadherins, and expressing stress fibers in a process known as endothelial mesenchymal transition (EndMT) [10]. The process of EndMT plays an important role in physiological angiogenic sprouting and also accounts for the fibroproliferative vasculopathy and fibrosis in multiple diseases including SSc [11]. These dynamic cell fate-switch processes make important contributions to fibrotic disease pathogenesis, and precise characterization of their underlying mechanisms opens new doors for targeted therapeutic interventions.

More recently, evidence has accumulated indicating that fibroblasts represent a functionally heterogeneous cell type, with distinct identities and states based on their embryonic origin, organ specificity, and localization within tissue $[12,13]$. The advent of single-cell RNA sequencing (scRNA-seq) has for the first time enabled the identification of multiple relevant and previously unidentified distinct fibroblast and myofibroblast subpopulations and states in a variety of normal tissue and fibrotic conditions $[14,15]$.

In the skin, lineage tracing studies in mice have identified multiple embryonically determined fibroblast lineages including one population of CD26+ cells with intrinsic profibrotic potential [16]. In addition to embryonic origin, the topographic location of fibroblasts also has a profound influence on their function. Skin fibroblasts located in the upper (papillary) or lower (reticular) layers of the dermis express different cell surface markers and contribute to fibrosis in distinct ways. Intriguingly, the cells in the lower reticular dermis correspond to cells that have been identified by having profibrotic potential and share a common mesenchymal precursor with the neighboring intradermal adipocytes [17]. In the lung, an analogous population of fibroblastic cells described as lipofibroblasts has been identified and shown to contribute to myofibroblast activation/formation in lung fibrosis [18]. In the liver, hepatic stellate cells, also known as lipocyte (Ito cells), are resident mesenchymal cells which play a major role in fibrosis by transdifferentiating into myofibroblasts during liver fibrosis [19].

Adipocytes are emerging as essential players in the pathogenesis of fibrosis. Adipocytes are mesenchymalderived cells which function not only as a storage organ for lipid droplets but also have important roles in endocrine and paracrine effects mediated through hormones known as adipokines [20]. It has become evident that changes in white adipose tissue modulate fibrotic outcomes in multiple organs [21]. In skin fibrosis, there is a consistent loss of dermal adipose tissue, obesity changes adipose homeostasis and promotes tissue fibrosis in multiple organs, excessive adipose tissue in the liver promotes the development of cirrhosis, and alterations in adipose in cancers including pancreatic and breast cancer can promote a fibrotic phenotype of the cancer-associated fibroblasts. Beyond these long-recognized but poorly appreciated clinical associations, a number of recent studies have uncovered an unexpected role of adipocytes as an important source of fibroblasts and myofibroblasts. The process whereby differentiated white adipocytes acquire a myofibroblast phenotype has been described as "adipocyte mesenchymal transition" or AMT. Cultured adipocytes under profibrotic stimuli including TGF- $\beta$ remarkably change morphology, downregulate their adipogenic genes/protein expression (PPAR- $\gamma$, perilipin, FABP4, etc.), and begin to express fibrotic genes/proteins (type I collagen, $\alpha$-SMA, etc.) indicating their transition to a myofibroblast phenotype [22・•] (Fig. 1). Importantly, there is now strong evidence that the AMT process is in fact bidirectional, and adipocytederived activated myofibroblasts can be made to regain a quiescent adipocytic phenotype $[23 \bullet \bullet, 24 \bullet \bullet]$. In light of the potential reversibility of AMT, this process therefore might represent a novel target of anti-fibrotic therapy. The rest of this review will explore the recent recognition and characterization of AMT and its implications in a variety of disease states. In addition to its emerging role in fibrosis, AMT may also play a key role in obesity, wound healing, and potentially in infectious processes including coronavirus (SARS-CoV-2) disease. 


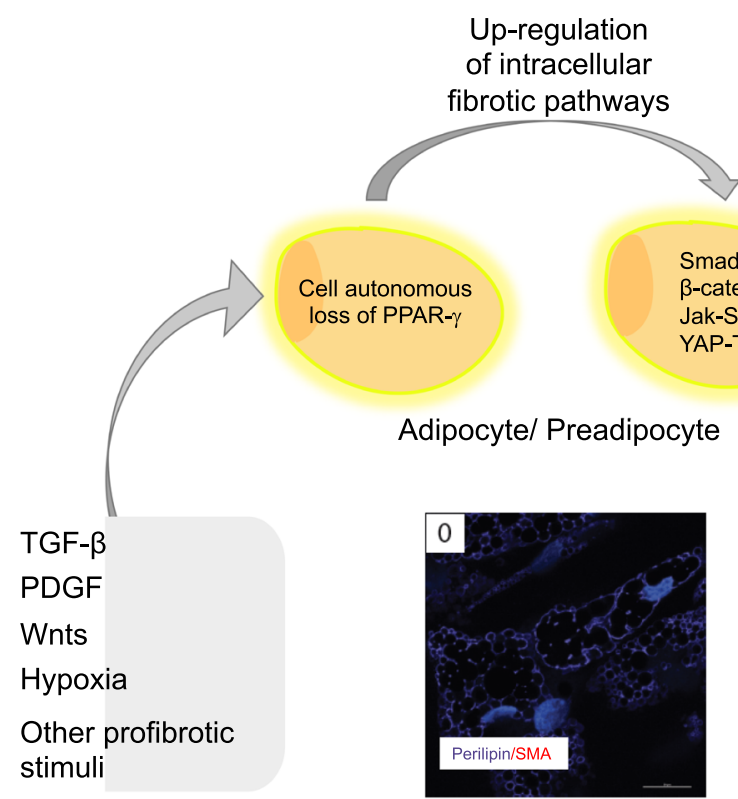

Fig. 1 Schematic of cellular mechanism of adipocyte-mesenchymal transition (AMT). In response to fibrotic stimuli (TGF- $\beta$, Wnts, Hypoxia, etc.), adipocytic cells downregulate adipogenic gene expression including PPAR- $\gamma$. In the absence of these signals, fibrotic pathways become predominant and lead this cell to undergo cellular reprogramming and change morphology. These cells can either transition to a preadipocyte/mesenchymal progenitor cell or directly to a

\section{AMT and Relevance to Disease: What Have We Learned}

\section{AMT and Skin Fibrosis}

Dermal white adipose tissue (dWAT) is a topographically distinct layer of white adipose tissue associated with the skin. While long recognized, dWAT was only recently described as an embryologically and functionally unique depot that is distinct from subcutaneous adipose tissue [25]. In mice, it can be clearly identified histologically by its localization between the reticular dermis and the striated panniculus carnosus muscle [25]. RNAseq experiments have also shown that dWAT is transcriptionally distinct from other white fat depots. In particular, compared with subcutaneous adipose tissue which is adjacent, dWAT expresses significantly more pro-inflammatory and immune-related genes [26••]. Across all adipose depots, dWAT exclusively expresses the gene cathelicidin antimicrobial peptide (Camp) [26••]. The important role of dWAT in skin biology has been largely ignored until recently [27]. Zhang et al. showed that Camp is produced in the skin during reactive adipogenesis in response to $S$. aureus intradermal injection, revealing dWAT's essential immune defense function [28]. dWAT is also dynamic in response to proadipogenic conditions including genetic adipose gain of function, high-fat diet, and treatment with PPAR- $\gamma$ agonists. Another important role of dWAT is related to hair follicle regeneration and wound healing $[29,30]$. During hair growth,
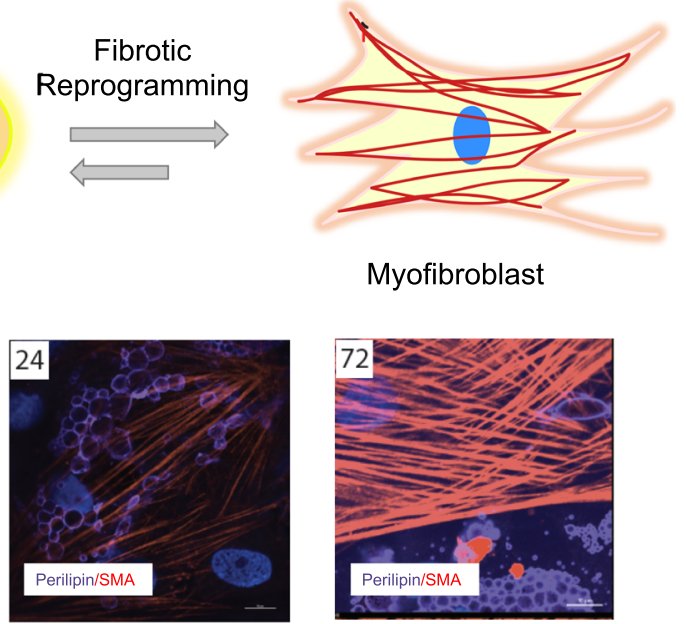

myofibroblast phenotype. This process is reversible in some circumstances. Below, adipocytes (marked by perilipin staining, purple) were treated with TGF- 3 . After $24 \mathrm{~h}$, these cells begin transitioning as marked by smooth muscle actin ( $\alpha$-SMA staining, red) in cells also expressing perilipin. By $72 \mathrm{~h}$, these cells have completed the transition to a myofibroblastic phenotype as marked by prominent expression of $\alpha$ SMA+-stress fibers with substantial loss of perilipin

hair follicles undergo cycling with temporally distinct phases: anagen, catagen, and telogen. In mice, dWAT expands during anagen and contracts during catagen. When adipocytes are absent or eliminated, normal hair cycling becomes dysregulated. Therefore, dwat homeostasis may contribute to the skin as well as hair integrity [26••]. Plikus et al. demonstrated that during wound healing, hair follicle regeneration in the center of the wound stimulates the reprogramming of myofibroblasts to adipocytes with subsequent decrease of pathologic scarring [23••]. Similar findings revealing the potential interaction between adipocytes and myofibroblasts have been shown ex vivo in keloids and hypertrophic scars [31].

Systemic sclerosis ( $\mathrm{SSc}$ ) is a chronic fibroinflammatory condition associated with fibrosis in the skin and multiple internal organs [32]. A distinguishing hallmark of SSc is the synchronous nature of extracutaneous multiple organ fibrosis. Skin fibrosis, the characteristic clinical manifestation of SSc, has long been recognized to be accompanied by loss of dWAT [33]. Biopsies from patients with SSc demonstrate decreased size and number of adipocytes, local macrophage infiltration, and replacement of dWAT with dense collagen (Fig. 2). Intriguingly, loss of dWAT is associated with and precedes the onset of skin fibrosis with replacement by collagen in multiple murine models [33, 34]. Lineage tracing and ex vivo differentiation experiments in mice demonstrated that intradermal adipocytes lose their specific markers (perilipin) and gain expression of myofibroblast markers 
Fig. 2 Loss of dermal white adipose tissue (dWAT) and replacement with collagen in systemic sclerosis (SSc). Skin biopsies from healthy control (left) and patient with diffuse cutaneous SSc and active disease (right) stained for H\&E. In contrast to control in which there are multiple areas of dense healthy adipocytic tissue, in SSc, there is almost complete loss of dWAT and replacement with fibrous matrix. Note smaller adipocytes (solid arrow) and remnants of adipocytes without nuclei (dashed arrow) surrounded by inflammatory infiltrate forming crown-like structures (arrowhead). Bars $=500 \mu \mathrm{m}$ (insets $=100 \mu \mathrm{m}$ )

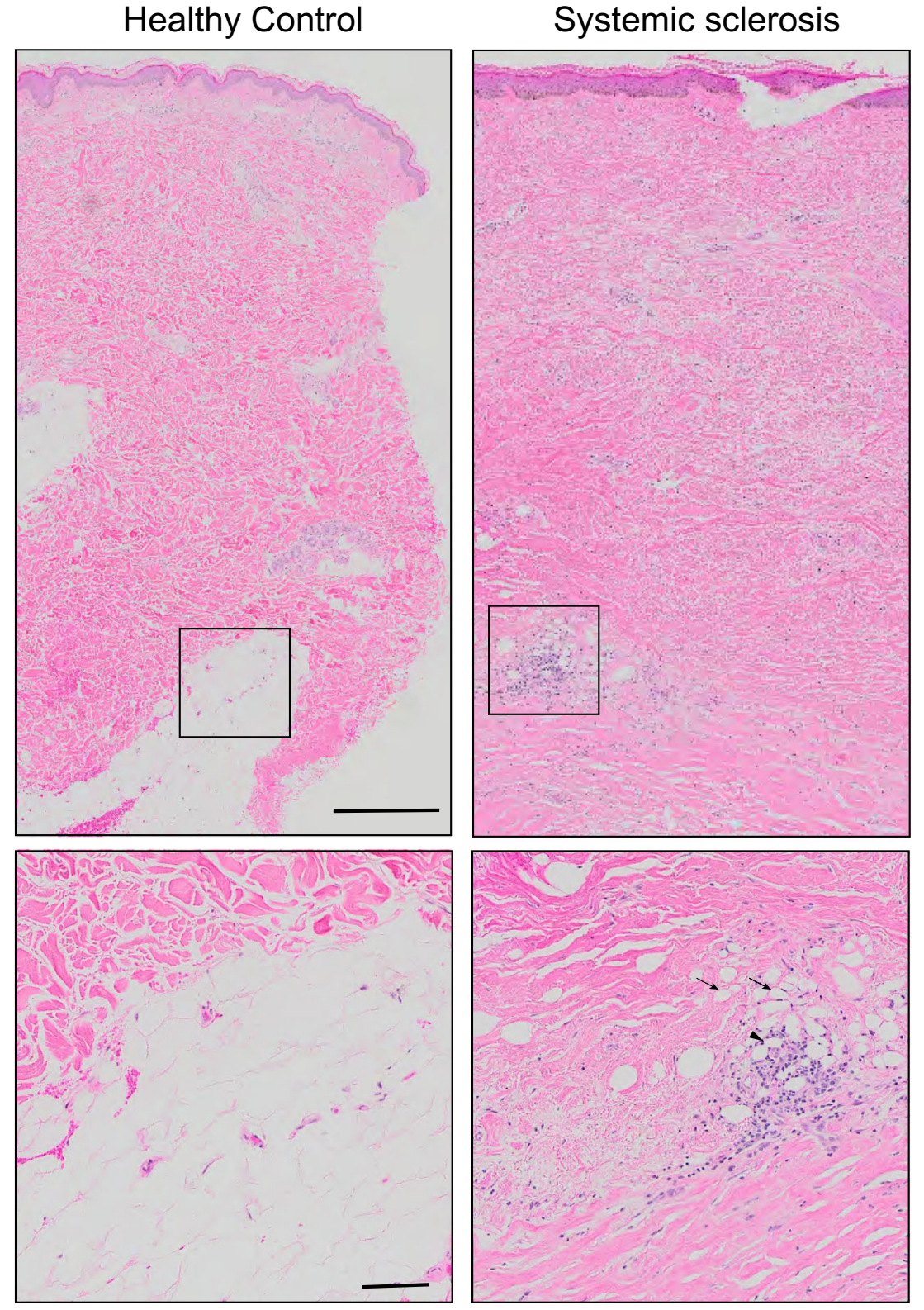

( $\alpha$-SMA) indicating they are undergoing a transition from adipocytes to myofibroblasts through AMT and contributing to the fibrotic phenotype [22••] (Fig. 3). More recently, Zhang et al. have replicated these findings using a doxycycline-inducible system which allowed tracing of mature adipocytes starting postnatally and with bleomycin, timed to avoid confounding by the hair cycle. Using this system, they confirmed that AMT occurs from mature adipocytes and likely represents a primary event in the pathogenesis of bleomycin-induced skin fibrosis [26••]. Moreover, using scRNA-seq, they identified that adipocytes first de-differentiate to $\operatorname{PDGFR} \alpha+$ preadipocytes as an intermediate step during AMT. Similarly, in androgenetic alopecia, it has been proposed that androgens can induce AMT leading to thickening of the follicular sheath and perifollicular fibrosis which limit the development of hair shafts [35].

\section{Lung Fibrosis}

In the lungs, lipofibroblasts represent a unique subpopulation of interstitial fibroblasts which contain lipid droplets and can secrete adipokines [36]. These unusual lung cells are located adjacent to type 2 alveolar epithelial cells and participate in surfactant production [37]. By using complementary lineagetracing strategies in a model of pulmonary fibrosis induced by bleomycin, El Agha et al. demonstrated that lipofibroblasts (adipose differentiation-related protein) contribute to the activated myofibroblast population (smooth-muscle actin) during lung injury through a process similar to AMT. Remarkably, 


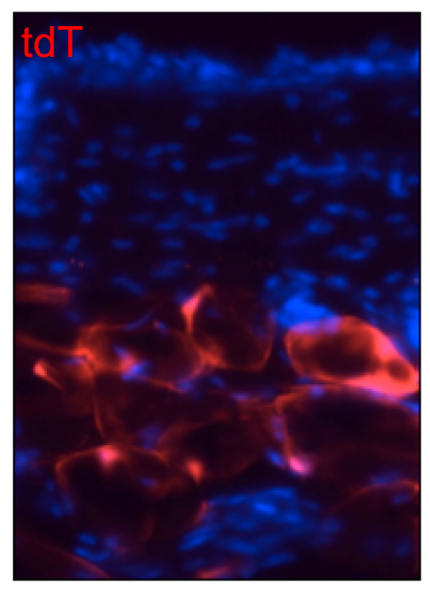

Adiponectin-Cre; tdT
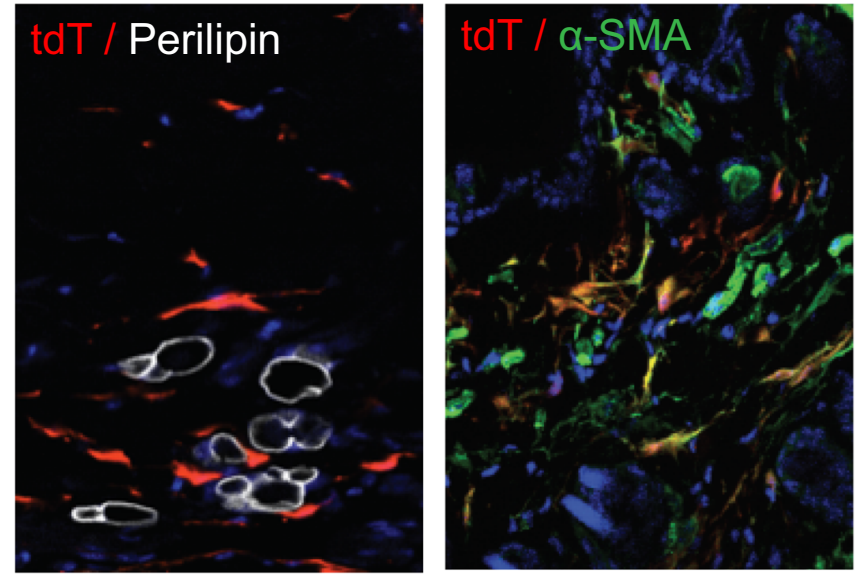

Adiponectin-Cre; tdT after bleomycin (day 21)
Fig. 3 Mature intradermal adipocytes undergo phenotypic transition to myofibroblast through adipocyte-mesenchymal transition (AMT). Adiponectin-Cre transgenic mice were crossed with Ai14 (tdTomato, tdT]) reporter mice to generate progeny expressing tdTomato fluorescent protein restricted to mature adipocytes (left panel). To trace the fate of intradermal adipocytes during fibrosis, mice were given

the authors showed that during fibrosis resolution, myofibroblasts de-differentiate back into lipofibroblasts suggesting that this is a dynamic process [24••]. This process can also be induced by metabolically active agents like metformin which have been shown to reverse the fibrotic phenotype of lung fibroblasts from patients with idiopathic pulmonary fibrosis (IPF) and bleomycin-induced lung fibrosis with the formation of adipocyte-like cells [38]. Moreover, the analysis of lung tissue from patients with IPF found a potential contribution of lipofibroblasts to human disease [24*0]. This phenomenon has been proposed in the pathogenic response to severe acute respiratory syndrome coronavirus 2 (SARS$\mathrm{CoV}-2$ ). SARS-CoV-2 strongly interacts with angiotensinconverting enzyme 2 (ACE2) for cell entry. ACE2 is normally expressed in the lungs by type 2 alveolar epithelial cells and also widely expressed in adipocytes. Obesity has been shown to be a major risk factor for a worse prognosis for SARS-CoV2 infection. This may be explained both by adipose tissue serving as a virus reservoir and by transdifferentiation of lipofibroblasts into myofibroblasts contributing to pulmonary fibrosis and the severity of lung involvement [39॰].

\section{Adipose Fibrosis}

Adipose tissue fibrosis is an important phenomenon associated with obesity which leads to dysfunctional adipose depots, inflammation, and endocrine abnormalities [40]. In a recent study, Marcelin et al. have identified PDGFR $\alpha+$ adipocyte progenitors in adipose tissues that can be classified on the basis of expressing CD9. CD $9^{\text {high }}$ preadipocytes are uniquely able to differentiate into subcutaneous injections of bleomycin (middle and right panels). Confocal imaging of tdT (red) and immunostained skin for perilipin (white) and $\alpha$-smooth muscle actin ( $\alpha$-SMA, green) demonstrated that tdT + cell change morphology from adipocyte to fibroblast, no longer costain with adipocyte marker perilipin and co-localize with myofibroblast marker $\alpha$-SMA. Hoechst 33342 (blue) counterstained

myofibroblasts and thus CD9 appears to mark a precursor cell population with profibrotic potential [41]. In order to understand the processes associated with these changes in obesity, Jones et al. evaluated the transcriptional response of adipocytes to a high-fat diet. The authors showed a profibrotic transcriptional reprogramming with upregulation of TGF- $\beta$ and Wnt signaling, as well as extracellular matrix-associated genes suggesting that adipocytes potentially transdifferentiate by AMT under these conditions [42•].

\section{Mechanisms Underlying AMT}

Multiple hypotheses regarding the cellular pathogenesis of AMT have been proposed, but a precise understanding of this important cellular reprogramming remains to be elucidated and is likely to be organ and disease specific. One hypothesis was that under cellular or systemic metabolic stress and/or hypoxia, adipocytes lose their proadipogenic regulators (i.e., PPAR- $\gamma$ ), driving their dedifferentiation into mesenchymal progenitor cells, which in turn under fibrotic stimuli (TGFß, Wnt, mechanical stress, etc.) differentiate into myofibroblasts. Alternatively, under stress/hypoxia, mature adipocytes may undergo apoptosis which would subsequently attract macrophages which could promote progenitors to differentiate into myofibroblasts. Finally, fibrotic stimuli may negatively regulate adipogenesis in mature adipocytes which then undergo transcriptional change and give rise to myofibroblasts. All of these reprogramming processes may occur with or without a change in adipokine secretion, which would function to 
further promote differentiation of adipocytes into myofibroblasts via endocrine mechanisms.

Lineage tracing studies in mice have shown that both transdifferentiation of mature adipocytes and their dedifferentiation to preadipocyte/progenitor cells are relevant contributors to myofibroblasts but only recently have been the major contributor been elucidated. Using lineage tracing systems, recent work using scRNA-seq has shown that mature intradermal adipocytes transdifferentiate into preadipocyte-like cells which expressed both markers indicating their previous state as mature adipocytes and expression of both the preadipocyte-specific gene $\operatorname{PDGFR} \alpha+$ and fibrogenic genes [26, 43••].

To further investigate the potentially distinct subsets of myofibroblasts during wound healing and fibrosis, Shook et al. performed flow cytometry analysis of the skin from mouse models of wound healing and fibrosis [43••]. Fibroblast-like cells were isolated on the basis of expression of PDGFR $\alpha, \mathrm{CD} 34$, and SCA1 which revealed four populations of cells CD29+CD34+, (adipocyte precursor), CD34+, CD29 $9^{\text {High }}, \mathrm{CD} 29^{\text {Low }}$. In skin from wounds, further analysis determined that only adipocyte precursor (CD29+CD34+) and CD29 ${ }^{\text {High }}$ could potentially become myofibroblasts. In fibrotic skin (induced by bleomycin), CD29 $9^{\text {High }}$ cells were the major population indicating that this population may play a key role in the pathogenesis of AMT. Remarkably, macrophage ablation significantly reduces the number of adipocyte precursor-derived myofibroblasts in wound healing. These macrophages were characterized and demonstrated surface expression of CD301b and secreted factors including plateletderived growth factor $\mathrm{C}$ and insulin-like growth factor 1 capable of stimulating proliferation of myofibroblasts. These findings suggest that therapies targeting specific myofibroblast and macrophage populations may represent a method of precisely treating impaired wound healing and fibrosis.

\section{Some Significant Unanswered Questions and Future Directions}

Adipocytes are remarkably pleiotropic mesenchymal cells that adopt multiple metabolic, immune, inflammatory, and homeostatic roles. Increasing evidence suggests that adipose tissue influences a variety of physiologic processes through paracrine effects [44]. Obesity is the most commonly encountered disorder of adipose tissue and has been shown to lead to a variety of deleterious outcomes including diabetes, hypercholesterolemia, cardiovascular disease and the metabolic syndrome, and adipose tissue fibrosis. Unlike healthy adipose tissue, adipocytes from obese individuals are under significant metabolic stress which leads them to an abnormal phenotype with features shared with AMT [42•]. Adipose stress is recognized to be present in a number of inflammatory, fibrotic, and neoplastic processes, and likely contribute to the role of AMT in these diseases. Adipocyte secretes adipokines as an endocrine organ with paracrine and anti-fibrotic effects. Adiponectin is a major adipokine which both regulates adipose tissue homeostasis and plays a role in preventing AMT.

As we discussed in this review, AMT is involved in multiple fibrotic diseases, wound healing, and obesityinduced adipose fibrosis. These findings should stimulate research in other diseases associated with adipose stress. In spondyloarthritis, fat metaplasia is associated with radiographic progression, and myofibroblasts have been shown to play a key role in syndesmophyte formation and ankylosis [45]. In rheumatoid arthritis and psoriatic arthritis, body habitus and adipokines contribute to disease severity and recent work has demonstrated a pathological role for specific synovial fibroblasts subsets [46]. Therefore, AMT may be an important phenomenon that should be further investigated and may represent a potential future targeted therapy. Likewise, AMT may play an important pathogenic role and should be further explored in a variety of diseases including non-alcoholic steatohepatitis, arrhythmogenic right ventricular dysplasia, kidney fibrosis, pancreatitis, and scarring skin diseases including keloid and discoid lupus.

The recent elucidation of the cell types and signaling pathways that drive AMT will be crucial in the development of therapies targeting fibrosis and wound healing. By specifically targeting subpopulations of macrophages, adipocytes and myofibroblasts with intrinsic profibrotic potential, therapies can be designed to remove pathogenic cells without compromising the otherwise important roles of these cells. Moreover, because AMT has been shown to be a dynamic reversible process, treatments which focus on dedifferentiating myofibroblasts back towards adipocytes/ progenitors may be rational in an established disease.

Acknowledgments We extend thanks to Warren G. Tourtellotte, Philipp E. Scherer, Theresa Lu, Valerie Horsley, and Jun Wei for their valuable insights. We also thank Maria Augusta Mackeldey for technical assistance.

Funding This work was supported by National Institute of Arthritis and Musculoskeletal and Skin Diseases Grant K08 AR070285 (to B.K.).

\section{Compliance with Ethical Standards}

Conflict of Interest The authors declare that they have no conflict of interest.

Human and Animal Rights and Informed Consent This article does not contain any studies with human or animal subjects performed by any of the authors. 


\section{References}

Papers of particular interest, published recently, have been highlighted as:

- Of importance

•- Of major importance

1. Rockey DC, Bell PD, Hill JA. Fibrosis-a common pathway to organ injury and failure. N Engl J Med. 2015;372(12):1138-49.

2. Kendall RT, Feghali-Bostwick CA. Fibroblasts in fibrosis: novel roles and mediators. Front Pharmacol. 2014;5:123.

3. Bejar R, Levine R, Ebert BL. Unraveling the molecular pathophysiology of myelodysplastic syndromes. J Clin Oncol. 2011;29(5): 504-15.

4. Santos A, Lagares D. Matrix stiffness: the conductor of organ fibrosis. Curr Rheumatol Rep. 2018;20(1):2.

5. Hinz B, Lagares D. Evasion of apoptosis by myofibroblasts: a hallmark of fibrotic diseases. Nat Rev Rheumatol. 2020;16(1):11-31.

6. Di Carlo SE, Peduto L. The perivascular origin of pathological fibroblasts. J Clin Invest. 2018;128(1):54-63.

7. Rosa I, Romano E, Fioretto BS, Manetti M. The contribution of mesenchymal transitions to the pathogenesis of systemic sclerosis. Eur J Rheumatol. 2019:1-8.

8. Nieto MA, Huang RY, Jackson RA, Thiery JP. Emt: 2016. Cell. 2016;166(1):21-45.

9. Distler JHW, Gyorfi AH, Ramanujam M, Whitfield ML, Konigshoff M, Lafyatis R. Shared and distinct mechanisms of fibrosis. Nat Rev Rheumatol. 2019;15(12):705-30.

10. Kovacic JC, Dimmeler S, Harvey RP, Finkel T, Aikawa E, Krenning G, et al. Endothelial to mesenchymal transition in cardiovascular disease: JACC state-of-the-art review. J Am Coll Cardiol. 2019;73(2):190-209.

11. Jimenez SA, Piera-Velazquez S. Endothelial to mesenchymal transition (EndoMT) in the pathogenesis of systemic sclerosisassociated pulmonary fibrosis and pulmonary arterial hypertension. Myth or reality? Matrix Biol. 2016;51:26-36.

12. Griffin MF, des Jardins-Park HE, Mascharak S, Borrelli MR, Longaker MT. Understanding the impact of fibroblast heterogeneity on skin fibrosis. Dis Model Mech. 2020;13(6).

13. Shaw TJ, Rognoni E. Dissecting fibroblast heterogeneity in health and fibrotic disease. Curr Rheumatol Rep. 2020;22(8):33.

14. Philippeos C, Telerman SB, Oules B, Pisco AO, Shaw TJ, Elgueta $\mathrm{R}$, et al. Spatial and single-cell transcriptional profiling identifies functionally distinct human dermal fibroblast subpopulations. J Invest Dermatol. 2018;138(4):811-25.

15. Lynch MD, Watt FM. Fibroblast heterogeneity: implications for human disease. J Clin Invest. 2018;128(1):26-35.

16. Rinkevich Y, Walmsley GG, Hu MS, Maan ZN, Newman AM, Drukker $\mathrm{M}$, et al. Skin fibrosis. Identification and isolation of a dermal lineage with intrinsic fibrogenic potential. Science. 2015;348(6232):aaa2151.

17. Driskell RR, Lichtenberger BM, Hoste E, Kretzschmar K, Simons $\mathrm{BD}$, Charalambous M, et al. Distinct fibroblast lineages determine dermal architecture in skin development and repair. Nature. 2013;504(7479):277-81.

18. El Agha E, Kramann R, Schneider RK, Li X, Seeger W, Humphreys BD, et al. Mesenchymal stem cells in fibrotic disease. Cell Stem Cell. 2017;21(2):166-77.

19. Higashi T, Friedman SL, Hoshida Y. Hepatic stellate cells as key target in liver fibrosis. Adv Drug Deliv Rev. 2017;121:27-42.

20. Zwick RK, Guerrero-Juarez CF, Horsley V, Plikus MV. Anatomical, physiological, and functional diversity of adipose tissue. Cell Metab. 2018;27(1):68-83.
21. Sun K, Tordjman J, Clement K, Scherer PE. Fibrosis and adipose tissue dysfunction. Cell Metab. 2013;18(4):470-7.

22.• Marangoni RG, Korman BD, Wei J, Wood TA, Graham LV, Whitfield ML, et al. Myofibroblasts in murine cutaneous fibrosis originate from adiponectin-positive intradermal progenitors. Arthritis Rheum. 2015;67(4):1062-73 A landmark study investigating the role of dWAT in skin fibrosis.

23.• Plikus MV, Guerrero-Juarez CF, Ito M, Li YR, Dedhia PH, Zheng $\mathrm{Y}$, et al. Regeneration of fat cells from myofibroblasts during wound healing. Science. 2017;355(6326):748-52 This study demonstrates the ability of dermal myofibroblasts to dedifferentiate into adipocytes during wound healing.

24.• El Agha E, Moiseenko A, Kheirollahi V, De Langhe S, Crnkovic S, Kwapiszewska G, et al. Two-way conversion between Lipogenic and myogenic fibroblastic phenotypes marks the progression and resolution of lung fibrosis. Cell Stem Cell. 2017;20(2):261-73 e3 An important study demonstrating plasticity of lipofibroblasts in fibrosis progression and resolution.

25. Driskell RR, Jahoda CA, Chuong CM, Watt FM, Horsley V. Defining dermal adipose tissue. Exp Dermatol. 2014;23(9):629-31.

26.• Zhang Z, Shao M, Hepler C, Zi Z, Zhao S, An YA, et al. Dermal adipose tissue has high plasticity and undergoes reversible dedifferentiation in mice. J Clin Invest. 2019;129(12):5327-42 This important study combines lineage tracing and transcriptomic analysis to characterize mature dermal adipocytes and explore their contribution in skin conditions including skin fibrosis.

27. Chen SX, Zhang LJ, Gallo RL. Dermal white adipose tissue: a newly recognized layer of skin innate defense. J Invest Dermatol. 2019;139(5):1002-9.

28. Zhang LJ, Guerrero-Juarez CF, Hata T, Bapat SP, Ramos R, Plikus $\mathrm{MV}$, et al. Innate immunity. Dermal adipocytes protect against invasive Staphylococcus aureus skin infection. Science. 2015;347(6217):67-71.

29. Festa E, Fretz J, Berry R, Schmidt B, Rodeheffer M, Horowitz M, et al. Adipocyte lineage cells contribute to the skin stem cell niche to drive hair cycling. Cell. 2011;146(5):761-71.

30. Schmidt BA, Horsley V. Intradermal adipocytes mediate fibroblast recruitment during skin wound healing. Development. 2013;140(7):1517-27.

31. Hoerst K, van den Broek L, Sachse C, Klein O, von Fritschen U, Gibbs S, et al. Regenerative potential of adipocytes in hypertrophic scars is mediated by myofibroblast reprogramming. J Mol Med (Berl). 2019;97(6):761-75.

32. Allanore Y, Simms R, Distler O, Trojanowska M, Pope J, Denton CP, et al. Systemic sclerosis. Nat Rev Dis Primers. 2015;1:15002.

33. Varga J, Marangoni RG. Systemic sclerosis in 2016: dermal white adipose tissue implicated in SSc pathogenesis. Nat Rev Rheumatol. 2017;13(2):71-2.

34. Marangoni RG, Lu TT. The roles of dermal white adipose tissue loss in scleroderma skin fibrosis. Curr Opin Rheumatol. 2017;29(6):585-90.

35. Kruglikov IL, Scherer PE. Adipocyte-myofibroblast transition as a possible pathophysiological step in androgenetic alopecia. Exp Dermatol. 2017;26(6):522-3.

36. Ahlbrecht $\mathrm{K}, \mathrm{McG}$ cowan SE. In search of the elusive lipofibroblast in human lungs. Am J Phys Lung Cell Mol Phys. 2014;307(8): L605-8.

37. Xie T, Wang Y, Deng N, Huang G, Taghavifar F, Geng Y, et al. Single-cell deconvolution of fibroblast heterogeneity in mouse pulmonary fibrosis. Cell Rep. 2018;22(13):3625-40.

38. Kheirollahi V, Wasnick RM, Biasin V, Vazquez-Armendariz AI, Chu X, Moiseenko A, et al. Metformin induces lipogenic differentiation in myofibroblasts to reverse lung fibrosis. Nat Commun. 2019;10(1):2987.

39. Kruglikov IL, Scherer PE. The role of adipocytes and adipocytelike cells in the severity of COVID-19 infections. Obesity (Silver 
Spring). 2020;28(7):1187-90 Proposes the provocative hypothesis that AMT can be implicated in the severity of COVID-19 lung pathology.

40. Khan T, Muise ES, Iyengar P, Wang ZV, Chandalia M, Abate N, et al. Metabolic dysregulation and adipose tissue fibrosis: role of collagen VI. Mol Cell Biol. 2009;29(6):1575-91.

41. Marcelin G, Ferreira A, Liu Y, Atlan M, Aron-Wisnewsky J, Pelloux V, et al. A PDGFR alpha-mediated switch toward CD9(high) adipocyte progenitors controls obesity-induced adipose tissue fibrosis. Cell Metab. 2017;25(3):673-85.

42. Jones JEC, Rabhi N, Orofino J, Gamini R, Perissi V, Vernochet C, et al. The adipocyte acquires a fibroblast-like transcriptional signature in response to a high fat diet. Sci Rep. 2020;10(1):2380 This study identifies that adipocytes in response to high-fat diet acquires a fibrogenic transcriptional profile.

43.• Shook BA, Wasko RR, Rivera-Gonzalez GC, Salazar-Gatzimas E, Lopez-Giraldez F, Dash BC, et al. Myofibroblast proliferation and heterogeneity are supported by macrophages during skin repair. Science. 2018;362(6417) This study identifies distinct subsets of myofibroblasts during wound healing and fibrosis and highlights an important interaction with a specific macrophage subpopulation.

44. Kahn CR, Wang G, Lee KY. Altered adipose tissue and adipocyte function in the pathogenesis of metabolic syndrome. J Clin Invest. 2019;129(10):3990-4000.

45. Beyer C, Distler JH. Changing paradigms in spondylarthritis: the myofibroblast signature. Arthritis Rheum. 2013;65(1):24-7.

46. Croft AP, Campos J, Jansen K, Turner JD, Marshall J, Attar M, et al. Distinct fibroblast subsets drive inflammation and damage in arthritis. Nature. 2019;570(7760):246-51.

Publisher's Note Springer Nature remains neutral with regard to jurisdictional claims in published maps and institutional affiliations. 